\title{
Chaotic Kabanov formula for the Azéma martingales
}

\author{
NICOLAS PRIVAULT ${ }^{1}$, JOSEP LLUÍS SOLE ${ }^{2 *}$ and JOSEP VIVES ${ }^{2 * *}$ \\ ${ }^{1}$ Département de Mathématiques, Université de la Rochelle, Avenue Marillac, 17042 La \\ Rochelle, Cedex 1, France.E-mail:nprivaul@univ-lr.fr \\ ${ }^{2}$ Departament de Mathemàtiques, Universitat Autònoma de Barcelona, 08193 Bellaterra, Spain. \\ E-mail:*jllsole@mat.uab.es; **vives@mat.uab.es
}

We derive the chaotic expansion of the product of $n$ th- and first-order multiple stochastic integrals with respect to certain normal martingales. This is done by application of the classical and quantum product formulae for multiple stochastic integrals. Our approach extends existing results on chaotic calculus for normal martingales and exhibits properties, relative to multiple stochastic integrals, polynomials and Wick products, that characterize the Wiener and Poisson processes.

Keywords: Azéma martingales; multiple stochastic integrals; product formulae

\section{Introduction}

The Wiener-Itô and Poisson-Itô chaotic decompositions give an isometric isomorphism between the Fock space $\Gamma\left(L^{2}\left(\mathbb{R}_{+}\right)\right)$and the space of square-integrable functionals of the process. This isomorphism is constructed by association of a symmetric function $f_{n} \in L^{2}\left(\mathbb{R}_{+}\right)^{\circ n}$ to its multiple stochastic integral. The isometry property comes from the fact that the angle brackets $\langle B\rangle_{t}$ and $\langle\tilde{N}\rangle_{t}$ of both the Wiener and compensated Poisson processes $\left(B_{t}\right)_{t \in \mathbb{R}_{+}},\left(\tilde{N}_{t}\right)_{t \in \mathbb{R}_{+}}$are equal to $t$. Therefore such isometric isomorphisms may exist for more general processes, provided their angle bracket equals $t$.

A martingale is said to be normal (Dellacherie et al. 1992) if its angle bracket is equal to $t$ and if the range of the multiple stochastic integrals isometry defined on $\Gamma\left(L^{2}\left(\mathbb{R}_{+}\right)\right)$is equal to the space of square-integrable functionals of this martingale. This last property is called the chaotic representation property.

The quadratic variation $\left([M, M]_{t}\right)_{t \in \mathbb{R}_{+}}$of a normal martingale $\left(M_{t}\right)_{t \in \mathbb{R}_{+}}$in $L^{4}(\Omega)$ with the chaotic representation property (or less restrictively, with the predictable representation property) satisfies the equation

$$
[M, M]_{t}=t+\int_{0}^{t} \phi_{s} \mathrm{~d} M_{s}, \quad t \in \mathbb{R}_{+},
$$

called a structure equation (Emery 1989), where $\left(\phi_{s}\right)_{s \in \mathbb{R}_{+}}$is a predictable process. On the other hand, a martingale satisfying (1) has angle bracket equal to $t$, but does not necessarily possess the chaotic representation property.

In this paper we work with solutions of such equations, which include the Wiener 
process, $\phi=0$; the compensated Poisson processes, $\phi$ constant and non-zero; and the Azéma martingales, $\phi_{t}=\beta M_{t}, \beta \in[-2,0[$.

Note that in the probabilistic framework of the structure equation one has to use a predictable version of $\phi$, which is $\phi_{t}=\beta M_{t^{-}}$in the case of the Azéma martingale. However, since we are working in $L^{2}$ with stochastic integrals with respect to a normal martingale, we do not need to distinguish between the adapted and predictable versions of $\phi$ (Dellacherie et al. 1992, p. 199). Thus, in what follows we will use $\phi_{t}=\beta M_{t}$ in the case of the Azéma martingale.

In the stochastic analysis on both the Wiener and Poisson spaces, multiplication formulae for multiple stochastic integrals proved to be useful tools as they give the chaotic expansion of the product of two multiple stochastic integrals. Recently (Russo and Vallois 1998), multiplication formulae have been proposed for multiple stochastic integrals with respect to normal martingales. However, these formulae do not give the explicit chaotic expansion of the considered products.

Our first goal in this paper is to relate the product formulae of Kabanov (1975), Russo and Vallois (1998) and Surgailis (1984) to their counterparts in quantum probability; see Section 3. With these tools we compute the chaotic expansion of the product of a multiple stochastic integral with a single stochastic integral, and refer to this formula as the chaotic Kabanov formula. This formula is proved for a class of martingales that includes the Azéma martingales; see Section 4. From the Kabanov formula, we have not obtained a general formula for the chaotic expansion of the product of two multiple stochastic integrals of orders $n$ and $m$ for this general class of martingales.

As an application of this Kabanov formula we show that a number of properties in stochastic analysis such as the possibility of expressing multiple stochastic integrals with polynomials and some properties of the Wick product are specific to the Wiener and Poisson cases; see Section 5. In Section 6 we consider formulae giving the derivation of a product.

\section{Notation and preliminaries}

We denote by

$$
\Gamma\left(L^{2}\left(\mathbb{R}_{+}\right)\right)=\bigoplus_{n \in \mathbb{N}} L^{2}\left(\mathbb{R}_{+}\right)^{\circ n}
$$

the symmetric Fock space over $L^{2}\left(\mathbb{R}_{+}\right)$, where $L^{2}\left(\mathbb{R}_{+}\right)^{\circ n}$ is endowed with the norm $\|\cdot\|_{L^{2}\left(\mathbb{R}_{+}\right) \circ n}^{2}=n !\|\cdot\|_{L^{2}\left(\mathbb{R}_{+}\right)^{\otimes n}}^{2}$, and $\langle\cdot, \cdot\rangle$ denotes the scalar product in $L^{2}\left(\mathbb{R}_{+}\right)$.

In this paper we work with a normal martingale $\left(M_{t}\right)_{t \in \mathbb{R}_{+}}$, with $M_{0}=0$, that satisfies the structure equation (1).

The multiple stochastic integral of a symmetric function $f_{n} \in L^{2}\left(\mathbb{R}_{+}\right)^{\circ n}$ with respect to $\left(M_{t}\right)_{t \in \mathbb{R}_{+}}$is denoted by

$$
I_{n}\left(f_{n}\right)=n ! \int_{0}^{\infty} \int_{0}^{t_{n}^{-}} \cdots \int_{0}^{t_{2}^{-}} f_{n}\left(t_{1}, \ldots, t_{n}\right) \mathrm{d} M_{t_{1}} \cdots \mathrm{d} M_{t_{n}},
$$


and as a convention we let

$$
I_{n}\left(f_{n}\right)=I_{n}\left(\tilde{f}_{n}\right)=I_{n}\left(f_{n}\left(t_{1}, \ldots, t_{n}\right)\right)
$$

if $f_{n} \in L^{2}\left(\mathbb{R}_{+}\right)^{\otimes n}$ is not symmetric, where $\tilde{f}_{n}$ denotes the symmetrization of $f_{n}$ in its $n$ variables.

We denote by $\mathscr{F}_{t}$ the $\sigma$-algebra generated by $\left\{M_{s}: 0 \leqslant s \leqslant t\right\}, t \in \mathbb{R}_{+}$.

We $\quad$ let $\quad \nabla^{-}: \Gamma\left(L^{2}\left(\mathbb{R}_{+}\right)\right) \rightarrow \Gamma\left(L^{2}\left(\mathbb{R}_{+}\right)\right) \otimes L^{2}\left(\mathbb{R}_{+}\right) \quad$ and $\quad \nabla^{+}: \Gamma\left(L^{2}\left(\mathbb{R}_{+}\right)\right) \otimes L^{2}\left(\mathbb{R}_{+}\right) \rightarrow$ $\Gamma\left(L^{2}\left(\mathbb{R}_{+}\right)\right)$denote the annihilation and creation operators on $\Gamma\left(L^{2}\left(\mathbb{R}_{+}\right)\right)$, defined as

$$
\nabla_{t}^{-} I_{n}\left(f_{n}\right)=n I_{n-1}\left(f_{n}(\cdot, t)\right), \quad t \in \mathbb{R}_{+}, \quad \nabla^{+}\left(I_{n}\left(f_{n+1}\right)\right)=I_{n+1}\left(f_{n+1}\right),
$$

where $f_{n+1}$ is symmetric in its $n$ first variables, $n \in \mathbb{N}$.

Let $\left.\mathscr{C}=\{] a, b]: a, b \in \mathbb{R}_{+}, a<b\right\}$. For $\left.\left.\left.] a, b\right],\right] c, d\right] \in \mathscr{A}$ we use the notation $\left.] a, b\right] \leqslant$ ]$c, d]$ if $b \leqslant c$. Let $\mathscr{S}_{n}, n \in \mathbb{N}$, denote the vector space

$$
\begin{aligned}
\mathscr{S}_{n}= & \left\{\sum_{i=1}^{m} \alpha_{i} I_{n}\left(1_{A_{1}^{i}} \circ \ldots \circ 1_{A_{n}^{i}}\right): A_{k}^{i} \cap A_{l}^{i}=\varnothing, 1 \leqslant k<l \leqslant n, A_{1}^{i}, \ldots, A_{n}^{i} \in \mathscr{C},\right. \\
& \left.\alpha_{i} \in \mathbb{R}, i=1, \ldots, m, m \geqslant 1\right\},
\end{aligned}
$$

and let $\mathscr{S}$ denote the vector space generated by $\cup_{n \in \mathbb{N}} \mathscr{S}_{n}$, which is dense in $L^{2}(\Omega)$.

Let $\mathscr{T}_{n}$ denote the vector space

$$
\begin{aligned}
\mathscr{T}_{n}= & \left\{\sum_{i=1}^{m} \alpha_{i} 1_{A_{1}^{i} I} \mathrm{n}\left(1 A_{1}^{i} \circ \ldots \circ 1_{A_{n}^{i}}\right): A_{k}^{i} \cap A_{l}^{i}=\varnothing, 1 \leqslant k<l \leqslant n,\right. \\
& \left.A_{0}^{k} \cap A_{0}^{l}=\varnothing, 1 \leqslant k<l \leqslant m, A_{0}^{i}, \ldots, A_{n}^{i} \in \mathscr{C l}, \alpha_{i} \in \mathbb{R}, i=1, \ldots, m, m \geqslant 1\right\} .
\end{aligned}
$$

We denote by $\mathscr{T}$ the vector space generated by $\cup_{n \geqslant 0} \mathscr{T}_{n}$, which is dense in $L^{2}(\mathrm{~d} \lambda)$ and in $L^{1}(\mathrm{~d} \lambda)$, where $\mathrm{d} \lambda=\mathrm{d} t \times \mathrm{d} P$.

Let $\mathscr{U}_{n}$ denote the vector space

$$
\begin{aligned}
\mathscr{U}_{n}= & \left\{\sum_{i=1}^{m} \alpha_{i} 1_{A_{0}^{i}} I_{n}\left(1_{A_{1}^{i}} \circ \cdots \circ 1_{A_{n}^{i}}\right): A_{k}^{i} \cap A_{l}^{i}=\varnothing, 0 \leqslant k<l \leqslant n,\right. \\
& \left.A_{0}^{k} \cap A_{0}^{l}=\varnothing, 1 \leqslant k<l \leqslant m, A_{0}^{i}, \ldots, A_{n}^{i} \in \mathscr{C}, \alpha_{i} \in \mathbb{R}, i=1, \ldots, m, m \geqslant 1\right\} .
\end{aligned}
$$

Note that $\mathscr{C}_{n}$ is made of processes in $\mathscr{T}_{n}$ that do not depend on the 'present'. We denote by $\mathscr{C}$ the vector space generated by $\cup_{n \geqslant 0} \mathscr{U}_{n}$, and by $\overline{\mathscr{C}}$ the completion of $\mathscr{C}$ in $L^{2}(\mathrm{~d} \lambda)$. The space $\overline{\mathscr{C}}$ is strictly smaller than $L^{2}(\mathrm{~d} \lambda)$ but it contains the adapted square-integrable processes and the range of $\nabla^{-}$, since $F \in \mathscr{S}$ implies $\nabla^{-} F \in \mathscr{U}$.

We will use the following lemma of Ma et al. (1998).

Lemma 1. Let $I_{n}\left(f_{n}\right), I_{1}\left(g_{1}\right) \in \mathscr{S}$. We have

$$
I_{n}\left(f_{n}\right) I_{1}\left(g_{1}\right)=I_{n+1}\left(f_{n} \circ g_{1}\right)+n \int_{0}^{\infty} I_{n-1}\left(f_{n}(\cdot, t)\right) g_{1}(t) \mathrm{d}[M, M]_{t} .
$$


As a consequence of this lemma, we have

$$
I_{n}\left(1_{A_{1}} \circ \cdots \circ 1_{A_{n}}\right)=I_{1}\left(1_{A_{1}}\right) \cdots I_{1}\left(1_{A_{n}}\right)
$$

whenever $A_{i} \cap A_{j}=\varnothing, 1 \leqslant i<j \leqslant n$.

We assume that there exists a set $\mathscr{P}$ of functionals dense in $L^{2}(\Omega)$ and included both in $L^{\infty}(\Omega)$ and in $\operatorname{Dom}\left(\nabla^{-}\right)$, and such that $\mathscr{P}$ is stable by $\nabla_{t}^{-}, t \in \mathbb{R}_{+}$. This assumption is satisfied in the cases of interest to us, that is in the Wiener and Poisson cases, and also in the case of the Azéma martingales since the latter is bounded (in this case it suffices to take $\mathscr{P}=\mathscr{S}$ ).

We recall the following identity, satisfied in general on Fock space:

$$
\mathrm{E}\left[\nabla^{+}(u)^{2}\right]=\|u\|_{L^{2}(\Omega) \otimes L^{2}\left(\mathbb{R}_{+}\right)}^{2}+\mathrm{E}\left[\int_{0}^{\infty} \int_{0}^{\infty} \nabla_{s}^{-} u_{t} \nabla_{t}^{-} u_{s} \mathrm{~d} s \mathrm{~d} t\right] .
$$

Definition 1. Let $\operatorname{Dom}_{1,2}\left(\nabla^{+}\right)$denote the set of processes $u \in L^{1}(\mathrm{~d} \lambda)$ such that there exists a sequence $\left(u_{n}\right)_{n \in \mathbb{N}} \subset \mathscr{T}$ converging in $L^{1}(\mathrm{~d} \lambda)$ to $u$ and such that $\left(\nabla^{+}\left(u_{n}\right)\right)_{n \in \mathbb{N}}$ converges in $L^{1}(\Omega)$ to an element of $L^{2}(\Omega)$ denoted as $\nabla^{+}(u)$.

Remark 1. The domain $\operatorname{Dom}_{1,2}\left(\nabla^{+}\right)$is well defined. We can show this as follows. Assume that $\left(u_{n}\right)_{n \in \mathbb{N}} \subset \mathscr{T}$ converges in $L^{1}(\mathrm{~d} \lambda)$ to 0 and that $\left(\nabla^{+}\left(u_{n}\right)\right)_{n \in \mathbb{N}}$ converges in $L^{1}(\Omega)$ to $G \in L^{2}(\Omega)$. From the duality relation

$$
\mathrm{E}\left[\nabla^{+}\left(u_{n}\right) F\right]=\mathrm{E}\left[\int_{0}^{\infty} u_{n}(t) \nabla_{t}^{-} F \mathrm{~d} t\right], \quad F \in \mathscr{P},
$$

we obtain $\mathrm{E}[G F]=0, F \in \mathscr{P}$, hence $G=0$ since $G \in L^{2}(\Omega)$.

This also means that if $u \in \operatorname{Dom}_{1,2}\left(\nabla^{+}\right)$and there exists a sequence $\left(u_{n}\right)_{n \in \mathbb{N}}$ converging in $L^{1}(\Omega)$ to $u$ and such that $\left(\nabla^{+}\left(u_{n}\right)\right)_{n \in \mathbb{N}}$ converges in $L^{1}(\Omega)$ to $G \in L^{2}(\Omega)$, then $G=\nabla^{+}(u)$. Naturally, $\operatorname{Dom}_{1,2}\left(\nabla^{+}\right)$contains the usual $L^{2}$ domain of $\nabla^{+}$which is denoted as $\operatorname{Dom}_{2}\left(\nabla^{+}\right)$.

Finally, let

$$
\begin{aligned}
\mathscr{T}_{n}= & \left\{\sum_{i=1}^{m} F_{i} 1_{] a_{i}, b_{i}\right]} I_{n}\left(1_{A_{1}^{i}} \circ \cdots \circ 1_{A_{n}^{i}}\right):\left[0, b_{i}\right] \cap A_{j}^{i}=\varnothing, j=1, \ldots, n,\right. \\
& \left.A_{k}^{i} \cap A_{l}^{i}=\varnothing, 1 \leqslant k<l \leqslant n, A_{1}^{i}, \ldots, A_{n}^{i} \in \mathscr{C}, F_{i} \in L^{2}\left(\mathscr{F}_{a_{i}}\right), i=1, \ldots, m, m \geqslant 1\right\},
\end{aligned}
$$

and $\mathscr{T}$ be the vector space generated by $\cup_{n} \mathscr{T}_{n}$. The space $\mathscr{T} \cap L^{2}(\mathrm{~d} \lambda)$ is not dense in $L^{2}(\mathrm{~d} \lambda)$, but it contains $\mathscr{C}$, and it is dense in $\overline{\mathscr{C}}$.

Remark 2. Let $u, v \in \mathscr{U}$, where $v$ is an adapted process. Then $u v \in \mathscr{T}$. To show this, we write $u=1_{A} I_{n}\left(1_{A_{1}} \circ \cdots \circ 1_{A_{n}}\right)$ with $A_{1}, \ldots, A_{k} \leqslant B$ and

$$
v=1_{B} I_{m}\left(1_{A_{n+1}} \circ \cdots \circ 1_{A_{n+m}}\right)
$$

with $A_{n+1}, \ldots, A_{n+m} \leqslant B$, since $v$ is adapted. Then 


$$
u v=1_{A \cap B} I_{k+m}\left(1_{A_{1}} \circ \cdots \circ 1_{A_{k}} \circ 1_{A_{n+1}} \circ \cdots \circ 1_{A_{n+m}}\right) I_{n-k}\left(1_{A_{k+1}} \circ \cdots \circ 1_{A_{n}}\right),
$$

hence $u v \in \mathscr{T}$.

Proposition 1. For $u \in \mathscr{T}$ such that $\int_{0}^{\infty} u_{t} \mathrm{~d} M_{t} \in L^{2}(\Omega)$, we have $u \in \operatorname{Dom}_{1,2}\left(\nabla^{+}\right)$and

$$
\nabla^{+}(u)=\int_{0}^{\infty} u_{t} \mathrm{~d} M_{t} \text {. }
$$

Proof. We start by choosing $u$ of the form $u=F 1_{] a, b]} I_{n}\left(1_{A_{1}} \circ \cdots \circ 1_{A_{n}}\right)$, with $F=$ $I_{m}\left(1_{B_{1}} \circ \cdots \circ 1_{B_{m}}\right), A_{i} \cap[0, b]=\varnothing, i=1, \ldots, n$, and $B_{1}, \ldots, B_{m} \subset[0, a]$. Then, from Lemma 1:

$$
\begin{aligned}
\nabla^{+}(u) & =I_{n+m+1}\left(1_{B_{1}} \circ \cdots \circ 1_{B_{m}} \circ 1_{] a, b]} \circ \cdots \circ 1_{A_{n}}\right) \\
& =I_{1}\left(1_{] a, b]}\right) I_{m}\left(1_{B_{1}} \circ \cdots \circ 1_{B_{m}}\right) I_{n}\left(1_{A_{1}} \circ \cdots \circ 1_{A_{n}}\right) \\
& =F I_{1}\left(1_{] a, b]}\right) I_{n}\left(1_{A_{1}} \circ \cdots \circ 1_{A_{n}}\right) .
\end{aligned}
$$

Hence, by linearity, for any $u \in \mathscr{T}$ and $F \in \mathscr{S}$,

$$
\nabla^{+}(u)=F I_{1}\left(1_{[a, b]}\right) I_{n}\left(1_{A_{1}} \circ \cdots \circ 1_{A_{n}}\right)=\int_{0}^{\infty} u_{t} \mathrm{~d} M_{t} .
$$

If $u \in \mathscr{T}$, then choosing a sequence $\left(F_{n}\right)_{n \in \mathbb{N}} \subset \mathscr{S}$ that converges in $L^{2}(\Omega)$ to $F$, and letting $u_{m}=F_{m} 1_{] a, b]} I_{n}\left(1_{A_{1}} \circ \cdots \circ 1_{A_{n}}\right)$, we have the convergence of $\left(u_{m}\right)_{m \in \mathbb{N}}$ in $L^{1}(\mathrm{~d} \lambda)$ to $u$ and of $\left(\nabla^{+}\left(u_{m}\right)\right)_{m \in \mathbb{N}}$ in $L^{1}(\Omega)$ to $\int_{0}^{\infty} u_{t} \mathrm{~d} M_{t}$ which belongs to $L^{2}(\Omega)$ by hypothesis.

\section{Classical and quantum product formulae}

The aim of this section is to link two versions of the product formula for a multiple stochastic integral and a single stochastic integral with respect to normal martingales.

The first one is called the Kabanov formula in reference to Kabanov (1975), which treated the Poisson case, $\phi=1$, and can be stated as follows. For $f_{n} \in L^{2}\left(\mathbb{R}_{+}\right)^{\circ n}$ and $g \in L^{2}([0, T])$, in particular $g$ with compact support, Lemma 1 was extended by Ma et al. (1998) and Russo and Vallois (1998) as

$$
I_{n}\left(f_{n}\right) I_{1}(g)=I_{n+1}\left(f_{n} \circ g\right)+n \int_{0}^{\infty} I_{n-1}^{*}\left(f_{n}(\cdot, t)\right) g(t) \mathrm{d}[M, M]_{t} .
$$

The second term in this formula is an integration over a diagonal, due to the Itô formula, and the notation ' $I_{n-1}^{*}\left(f_{n}(\cdot, t)\right)$ ' will be made precise in Definition 2.

The second product formula uses only chaos expansions and Fock space, and can be found in the work of quantum probabilists. It is often stated in the formalism of quantum stochastic integrals: 


$$
\int_{0}^{\infty} g_{t} \mathrm{~d} M_{t}=\int_{0}^{\infty} g_{t} \mathrm{~d} a_{t}^{+}+\int_{0}^{\infty} g_{t} \mathrm{~d} a_{t}^{-}+\int_{0}^{\infty} \phi_{t} g_{t} \mathrm{~d} a_{t}^{\circ} ;
$$

cf. Parthasarathy (1990) and Biane (1995, Proposition 18).

The definition of quantum stochastic integrals as operators poses several functional analytic problems. See the paper of Attal and Lindsay (1997) for recent extensions of their definition. In general, the above relation does not hold in $L^{2}(\Omega)$ except in a weak sense (Biane 1995), where this formula is proved for $F$ an exponential vector, and for bounded predictable $g$ and $\phi$, with compact support.

Relation (7) can be reformulated formally using the operators $\nabla^{-}, \nabla^{+}$, as follows:

$$
F \nabla^{+}(g)=\nabla^{+}(F \otimes g)+\int_{0}^{\infty} g(t) \nabla_{t}^{-} F \mathrm{~d} t+\nabla^{+}\left(g \phi \nabla^{-} F\right)
$$

(see, for example, Attal 1998; Biane, 1995; and the references therein), the only difference between (7) and (8) being a change of notation.

In the Poisson case, this formula also appears in the papers of Nualart and Vives (1990) and Dermoune et al. (1988). It will play an important role in the computation of the chaotic expansion of the Kabanov formula, since unlike (3) and (6) it uniquely involves calculations on chaos.

The product formula (8) can be rewritten in the language of quantum stochastic differentials as

$$
\int_{0}^{\infty} g(t) \mathrm{d} M_{t}=\int_{0}^{\infty} g(s) \mathrm{d} B_{s}+\int_{0}^{\infty} \phi(s) g(s) \mathrm{d} a_{t}^{\circ},
$$

or

$$
\mathrm{d} M_{t}=\mathrm{d} B_{t}+\phi(t) \mathrm{d} a_{t}^{\circ} .
$$

In the Wiener interpretation of the Fock space, the differential operator $\mathrm{d} B_{t}$ identifies with multiplication by the classical Brownian differential, and this formula states that the differential operator $\mathrm{d} M_{t}=\mathrm{d} B_{t}+\phi(t) \mathrm{d} a_{t}^{\circ}$ is identified with the multiplication operator by the classical differential $\mathrm{d} M_{t}$ when the Fock space is identified with the $L^{2}$ space of $\left(M_{t}\right)_{t \in \mathbb{R}_{+}}$. However, equation (9) has no classical interpretation because the operator processes $\left(B_{t}\right)_{t \in \mathbb{R}_{+}}$and $\left(a_{t}^{\circ}\right)_{t \in \mathbb{R}_{+}}$cannot be interpreted simultaneously as multiplication operators in the same probabilistic interpretation of the Fock space (the reason for this is that they do not commute). Consequently, (9) does not have a classical meaning; it only defines an operator process on Fock space.

If $\phi(t)$ is a function of $M_{t}$, for example, $\phi(t)=f\left(M_{t}\right)$, then (9) becomes a quantum stochastic differential equation, in the space of operators on Fock space,

$$
\mathrm{d} M_{t}=\mathrm{d} B_{t}+f\left(M_{t}\right) \mathrm{d} a_{t}^{\circ},
$$

which does not have a classical interpretation, whatever the interpretation chosen for the Fock space.

In order to find the multiplication formula for multiple stochastic integrals with respect to $\left(M_{t}\right)_{t \in \mathbb{R}_{+}}$, one has to compute in particular the chaos expansion of $M_{t}$ multiplied by a multiple stochastic integral. This means that the explicit expression of $M_{t}$ as an operator on 
Fock space has to be obtained. A way to obtain this expression is to solve (10) in the space of operators on Fock space, that is to determine the process of operators associated with $\left(M_{t}\right)_{t \in \mathbb{R}_{+}}$. The simplest case is the linear case, $f(x)=\beta x, \beta \in[-2,0[$, which corresponds to the family of Azéma martingales. In this case, (10) reads

$$
\mathrm{d} M_{t}=\mathrm{d} B_{t}+\beta M_{t} \mathrm{~d} a_{t}^{\circ},
$$

which can be formally solved as a linear equation. It can be easily shown that its solution is

$$
M_{t}=\sum_{n \geqslant 0} \beta^{n} \int_{0}^{t} \int_{0}^{t_{n-1}} \cdots \int_{0}^{t_{2}} B_{t_{1}} \mathrm{~d} a_{t_{2}}^{\circ} \cdots \mathrm{d} a_{t_{n-1}}^{\circ},
$$

or

$$
M_{t}=\sum_{n \geqslant 0} \beta^{n} \int_{0}^{t} \int_{0}^{t_{n}} \cdots \int_{0}^{t_{1}} \mathrm{~d} B_{t_{0}} \mathrm{~d} a_{t_{1}}^{\circ} \cdots \mathrm{d} a_{t_{n}}^{\circ} .
$$

This expression can now be rewritten explicitly in terms of operators on Fock space:

$$
\begin{aligned}
M_{t} F= & \sum_{n \geqslant 0} \beta^{n} \nabla^{+}\left(1_{[0, t]}\left(\nabla^{+}\left(\cdots \nabla^{+}\left(\int_{0}^{\cdot} \nabla_{s}^{-} \mathrm{d} s \nabla^{-} \cdots \nabla^{-} F\right)\right)\right)\right) \\
& +\sum_{n \geqslant 0} \beta^{n} \nabla^{+}\left(1_{[0, t]}\left(\nabla^{+}\left(\cdots \nabla^{+}\left(\nabla^{+}\left(1_{[0, \cdot]} \nabla^{-} \cdots \nabla^{-} F\right)\right)\right)\right)\right) .
\end{aligned}
$$

When $F=I_{n}\left(f_{n}\right)$, this gives the explicit chaos expansion of $M_{t} I_{n}\left(f_{n}\right)$.

The above calculation is formal and some further computations are required in order to obtain the explicit chaos expansion of $M_{t} I_{n}\left(f_{n}\right)$. In this paper we justify each of the above steps by explicit calculations and carry out the final computations suggested in (12). The aim of this remark was to show that the quantum stochastic point of view gives a different understanding of the problem and provides a quick solution by reducing the problem to the determination of a quantum diffusion. We stress that although the solution process has a classical version, the diffusion equation (10) is meaningful only in the space of operators on Fock space. Also, those remarks show that the reason why explicit calculation can be carried out is in fact that (11) is a linear equation.

We also note that a slightly more general case can be considered, $\phi(t)=$ $\lambda_{t}+\int_{0}^{t} \beta_{t}(s) \mathrm{d} M_{s}$, which is still linear. In this case the solution reads

$$
\begin{aligned}
M_{t}= & \sum_{n \geqslant 0} \int_{0}^{t} \int_{0}^{t_{n}} \beta_{t_{n}}\left(t_{n-1}\right) \int_{0}^{t_{n-1}} \cdots \int_{0}^{t_{1}} \mathrm{~d} B_{t_{0}} \mathrm{~d} a_{t_{1}}^{\circ} \cdots \mathrm{d} a_{t_{n}}^{\circ} \\
& +\sum_{n \geqslant 0} \int_{0}^{t} \int_{0}^{t_{n}} \beta_{t_{n}}\left(t_{n-1}\right) \int_{0}^{t_{n-1}} \cdots \int_{0}^{t_{2}} \beta_{t_{2}}\left(t_{1}\right) \lambda_{t_{1}} \mathrm{~d} a_{t_{0}}^{\circ} \cdots \mathrm{d} a_{t_{n}}^{\circ} .
\end{aligned}
$$

This situation is considered in our paper.

Our aim in this section is to prove $(7)$ in $L^{2}(\Omega)$ from (6). The proof of Biane (1995) see also Attal (1998) - relies on the construction of quantum stochastic calculus, whereas 
our proof uses more classical probabilistic arguments. We also prove that $(8)$ holds in $L^{2}$ under assumptions that are satisfied in our setting.

Definition 2. Following Ma et al. (1998) and Russo and Vallois (1998), let $\mu$ denote the measure on $\mathbb{R}_{+} \times \Omega$ defined as $\mu([0, t] \times A)=\mathrm{E}\left[1_{A}[M, M]_{t}\right], A \in \mathscr{F}, t \in \mathbb{R}_{+}$, and let $v=(\lambda+\mu) / 2$. For $u \in \overline{\mathscr{C}}$ we denote by $u^{*}$ the limit in $L^{2}(\mathrm{~d} v)$ of any sequence $\left(u_{n}\right)_{n \in \mathbb{N}} \subset \mathscr{Q b}$ that converges in $L^{2}(\mathrm{~d} \lambda)$ to $u$.

Note that $u=u^{*} \lambda$-a.e., but not $\mu$-a.e., except in the Wiener case $(\phi=0)$, and in general for $u \in \mathscr{U}$. From Lemma 5.2. of Ma et al. (1998) we have

$$
\|u\|_{L^{2}(\mathrm{~d} \lambda)}=\left\|u^{*}\right\|_{L^{2}(d \mu)}, \quad u \in \overline{\mathscr{b}}
$$

In what follows we fix $T>0$. For $u \in \overline{\mathscr{C}}, \int_{0}^{T} u_{t}^{*} \mathrm{~d}[M, M]_{t}$ is defined a.e. as an integral with respect to an increasing process and it belongs to $L^{1}(\Omega)$, since

$$
\mathrm{E}\left[\left|\int_{0}^{T} u_{t}^{*} \mathrm{~d}[M, M]_{t}\right|\right] \leqslant T^{1 / 2}\left\|u^{*}\right\|_{L^{2}(\mathrm{~d} \mu)} \leqslant T^{1 / 2}\|u\|_{L^{2}(\mathrm{~d} \lambda)} .
$$

The following lemma allows us to prove the quantum product formula (7) from the probabilistic product formula (6).

Lemma 2. Let $u \in \overline{\mathscr{C}}$ and assume that $1_{[0, T]} \phi \in L^{2}(\mathrm{~d} \lambda)$ for some $T \in \mathbb{R}_{+}$. Then $1_{[0, T]} u \phi \in \operatorname{Dom}_{1,2}\left(\nabla^{+}\right)$if and only if

$$
\int_{0}^{T} u_{t}^{*} \mathrm{~d}[M, M]_{t} \in L^{2}(\Omega)
$$

and in this case,

$$
\int_{0}^{T} u_{t} \mathrm{~d} t+\nabla^{+}\left(1_{[0, T]} u \phi\right)=\int_{0}^{T} u_{t}^{*} \mathrm{~d}[M, M]_{t} .
$$

Proof. We start by assuming that $u \in \mathscr{C}$ is of the form $\left.u=\sum_{i=1}^{n} F_{i} 1_{]} a_{i}, b_{i}\right]$, where $b_{i} \leqslant T$. Since $\phi$ is adapted we can choose a sequence $\left(v_{n}\right)_{n \in \mathbb{N}} \subset \mathscr{U}$ of adapted processes converging to $\phi 1_{[0, T]}$ in $L^{2}(\mathrm{~d} \lambda)$. Then, from Remark $2, u v_{n} \in \mathscr{T}$ and, from Proposition 1 ,

$$
\left(\nabla^{+}\left(u v_{n}\right)\right)_{n \in \mathbb{N}}=\left(\int_{0}^{\infty} u v_{n}(t) \mathrm{d} M_{t}\right)_{n \in \mathbb{N}} .
$$

Hence $\left(\nabla^{+}\left(u v_{n}\right)\right)_{n \in \mathbb{N}}$ converges in $L^{1}(\Omega)$ to

$$
\sum_{i=1}^{n} F_{i} \int_{a_{i}}^{b_{i}} \phi_{s} \mathrm{~d} M_{s}=\int_{0}^{T} u_{t} \phi_{t} \mathrm{~d} M_{t} \in L^{2}(\Omega),
$$

$u \phi \in \operatorname{Dom}_{1,2}\left(\nabla^{+}\right)$and $\nabla^{+}(u \phi)=\int_{0}^{T} u_{t} \phi_{t} \mathrm{~d} M_{t}$, that is, 


$$
\int_{0}^{T} u_{t} \mathrm{~d} t+\nabla^{+}(u \phi)=\int_{0}^{T} u_{t} \mathrm{~d}[M, M]_{t} .
$$

If $u \in \overline{\mathscr{C}}$ we approximate it in $L^{2}(\mathrm{~d} \lambda)$ by a sequence $\left(u_{n}\right)_{n \in \mathbb{N}}$ in $\mathscr{\mathscr { C }}$. Then, from (13) we have that $\left(\int_{0}^{T} u_{n}(t) \mathrm{d} t+\nabla^{+}\left(u_{n} \phi\right)\right)_{n \in \mathbb{N}}=\left(\int_{0}^{T} u_{n}(t) \mathrm{d}[M, M]_{t}\right)_{n \in \mathbb{N}}$ converges in $L^{1}(\Omega)$ to $\int_{0}^{T} u^{*} \mathrm{~d}[M, M]_{t}$.

We now prove the quantum product formula (7), under assumptions different from that of Biane (1995).

Proposition 2. Let $F$ be in a finite sum of chaos and let $h \in L^{2}([0, T])$ be bounded. Then $h \phi \nabla^{-} F \in \operatorname{Dom}_{1,2}\left(\nabla^{+}\right)$if and only if $I_{1}(h) F \in L^{2}(\Omega)$ and in this case,

$$
I_{1}(h) F=\nabla^{+}(h \otimes F)+\left(h, \nabla^{-} F\right)_{L^{2}\left(\mathbb{R}_{+}\right)}+\nabla^{+}\left(h \phi \nabla^{-} F\right) .
$$

Proof. We let $F=I_{n}\left(f_{n}\right) \in \mathscr{S}, \quad h \in L^{2}([0, T])$, and apply (6) and Lemma 2 to $h \nabla^{-} I_{n}\left(f_{n}\right) \in \overline{\mathscr{C}}$, since $\overline{\mathscr{C}}$ contains the range of $\nabla^{-}$. In the general case we choose a sequence $\left(F_{n}\right)_{n \in \mathbb{N}} \subset \mathscr{S}$ that converges to $F$ in $L^{2}(\Omega)$. The right-hand side of (15) converges to $\nabla^{+}(h \otimes F)+\int_{0}^{T} h_{t} \nabla_{t}^{-} F \mathrm{~d} t+\nabla^{+}\left(h \phi \nabla^{-} F\right)=I_{1}(h) F \quad$ in $L^{1}(\Omega)$ and $\left(h \phi \nabla^{-} F_{n}\right)_{n \in \mathbb{N}}$ converges to $h \phi \nabla^{-} F$ in $L^{1}(\Omega)$ as $n$ goes to infinity. Hence $h \phi \nabla^{-} F \in \operatorname{Dom}_{1,2}\left(\nabla^{+}\right)$if and only if $I_{1}(h) F \in L^{2}(\Omega)$.

The result extends to bounded simple adapted processes of the form $h=G 1_{\left[t_{1}, t_{2}[\right.}$ for $\mathscr{F}_{t_{1}}$-measurable $G$, since in this case $\nabla_{s}^{-} G=0, s>t_{1}$ (see Lemma 4.1. of Ma et al. 1998), which means that $\nabla^{+}\left(1_{\left[t_{1}, t_{2}[\right.} G\right)=G \nabla^{+}\left(1_{\left[t_{1}, t_{2}[\right.}\right)$.

We close this section with a remark on the link between independence of stochastic integrals and their deterministic kernels. The above formula easily gives information on the chaotic expansion of a product of stochastic integrals, for general $\phi$. If $f, g \in L^{2}([0, T])$ are such that $f g \phi \in \operatorname{Dom}_{1,2}\left(\nabla^{+}\right)$, then

$$
I_{1}(f) I_{1}(g)=I_{2}(f \circ g)+(f, g)_{L^{2}\left(\mathbb{R}_{+}\right)}+\nabla^{+}(f g \phi) .
$$

Letting $T$ go to infinity, the formula holds for $f, g \in L^{2}\left(\mathbb{R}_{+}\right)$, provided $f g \phi \in \operatorname{Dom}_{1,2}\left(\nabla^{+}\right)$. From this formula it is clearly seen that the chaotic expansion of $I_{1}(f) I_{1}(g)$ may be an infinite sum of multiple stochastic integrals, depending on the chaotic expansion of $\phi$. This formula can be applied in order to obtain a necessary condition for the independence of stochastic integrals. The result of Urbanik (1967) says that if a stochastic process $\left(X_{t}\right)_{t \in \mathbb{R}_{+}}$has stationary and independent increments then independence of $\int_{0}^{\infty} f_{t} \mathrm{~d} X_{t}$ and $\int_{0}^{\infty} g_{t} \mathrm{~d} X_{t}$ implies $f g=0$, except if $X$ is Gaussian, in which case the condition becomes $(f, g)_{L^{2}\left(\mathbb{R}_{+}\right)}=0$. In the Gaussian case this property has been further extended to multiple stochastic integrals by Üstünel and Zakai (1990). See also Privault (1996) for the case of deterministic $\phi$. We can now give an extension of this property to more general $\phi$.

Proposition 3. Assume that the chaotic expansion of $\phi_{t}$ does not contain terms of order 1. Then, independence of $I_{1}(f)$ and $I_{1}(g)$ implies $(f, g)_{L^{2}\left(\mathbb{R}_{+}\right)}=0$ and $f_{t} g_{t}=0, \mathrm{E}\left[\phi_{t}^{2}\right] \mathrm{d} t$-a.e. 
Proof. We follow an argument of Üstünel and Zakai (1990) that deals with the Wiener case.

$$
\begin{aligned}
\|f \circ g\|_{L^{2}\left(\mathbb{R}_{+}\right)^{\circ 2}}^{2} & \geqslant(f, f)_{L^{2}\left(\mathbb{R}_{+}\right)}(g, g)_{L^{2}\left(\mathbb{R}_{+}\right)}=\mathrm{E}\left[I_{1}(f)^{2}\right] \mathrm{E}\left[I_{1}(g)^{2}\right] \\
& =\mathrm{E}\left[\left(I_{1}(f) I_{1}(g)\right)^{2}\right] \\
& =\|f \circ g\|_{L^{2}\left(\mathbb{R}_{+}\right)^{\circ 2}}^{2}+(f, g)_{L^{2}\left(\mathbb{R}_{+}\right)}^{2}+\left\|\nabla^{+}(f g \phi)\right\|_{L^{2}(\Omega)}^{2},
\end{aligned}
$$

where we used the assumption on the chaotic expansion of $\phi$ which implies the orthogonality of $\nabla^{+}(f g \phi)$ and $I_{2}(f \circ g)$ in (16). Hence $(f, g)_{L^{2}\left(\mathbb{R}_{+}\right)}=0$, and $\nabla^{+}(f g \phi)=0$ a.e. Hence $f g \phi=0, \mathrm{~d} \lambda$-a.e.

Note that the hypothesis of this proposition do not include the Azéma martingales.

\section{Chaotic Kabanov formula}

The purpose of this section is to obtain in Theorem 1 a chaotic formula for the product $I_{1}(g) I_{n}\left(f_{n}\right)$ using Proposition 2 applied to $F=I_{n}\left(f_{n}\right)$. Under certain assumptions on $f_{n}$ and $g$, we have

$$
I_{1}(g) I_{n}\left(f_{n}\right)=I_{n+1}\left(f_{n} \circ g\right)+n I_{n-1}\left(\left\langle f_{n}(*, \cdot), g(\cdot)\right\rangle\right)+n \nabla^{+}\left(g(\cdot) \phi(\cdot) I_{n-1}\left(f_{n}(*, \cdot)\right)\right) .
$$

If $\phi$ is deterministic, in particular in the Wiener and Poisson cases, this formula easily yields the Kabanov formula (Privault 1996). On the other hand, if $\phi$ is random the chaos expansion of the term $\phi \cdot I_{n-1}\left(f_{n}(*, \cdot)\right)$ is unknown unless $\phi_{t}$ belongs to the first chaos, i.e. $\phi(t)=\alpha(t)+I_{1}\left(\beta_{t}\right), t \geqslant 0$, since in this case an induction argument can be used in (17) to compute the term $\phi \cdot I_{n-1}\left(f_{n}\left(t_{1}, \ldots, t_{n-1}, \cdot\right)\right)$ and to determine the chaotic expansion of the product $I_{1}\left(\beta_{t}\right) I_{n}\left(f_{n}\right)$. Thus in this case the chaotic expansion of $I_{1}(g) I_{n}\left(f_{n}\right)$ can be obtained as a consequence of Proposition 2.

In this section, we assume that $\phi$ is of the form $\phi(t)=\alpha(t)+I_{1}\left(\beta_{\mathrm{t}}\right), t \geqslant 0$, where $\alpha$ is a locally bounded function from $\mathbb{R}_{+}$to $\mathbb{R}$ and $\beta_{t}(\cdot)$ is a bounded function from $\mathbb{R}_{+}$to $\mathbb{R}$ with support in $[0, t]$, for any fixed $t \in \mathbb{R}_{+}$. This situation is more general than the situation of Russo and Vallois (1998, Section 4); moreover, chaotic expansions are completely determined here.

We have the following lemma:

Lemma 3. Let $\theta \in \mathbb{R}_{+}$. Let $f_{p} \in L^{2}\left(\mathbb{R}_{+}\right)^{\circ p}$ and

$$
\zeta\left(t_{1}, \ldots, t_{k-1} ; t_{k}\right)=\beta_{t_{2}}\left(t_{1}\right) \cdots \beta_{t_{k}}\left(t_{k-1}\right), \quad t_{1}, \ldots, t_{k} \in \mathbb{R}_{+}, \quad k>1,
$$

and $\zeta(t)=1, t \in \mathbb{R}_{+}$, for $k=1$. The chaos expansion of the product $I_{1}\left(\beta_{\theta}\right) I_{p}\left(f_{p}\right)$ is given by

$$
I_{1}\left(\beta_{\theta}\right) I_{p}\left(f_{p}\right)=I_{p+1}\left(g_{p+1}^{+}(* ; \theta)\right)+I_{p}\left(g_{p}^{\circ}(* ; \theta)\right)+I_{p-1}\left(g_{p-1}^{-}(* ; \theta)\right),
$$

where 


$$
g_{p+1}^{+}\left(t_{1}, \ldots, t_{p+1} ; \theta\right)=\sum_{j=1}^{p+1} \frac{p !}{(j-1) !} \zeta\left(t_{j}, \ldots, t_{p+1} ; \theta\right) f_{p}\left(t_{1}, \ldots, \hat{t}_{j}, \ldots, t_{p+1}\right),
$$

where $\hat{t}_{j}$ means that $t_{j}$ is omitted in the arguments of $f_{p}$,

$$
g_{p}^{\circ}\left(t_{1}, \ldots, t_{p} ; \theta\right)=f_{p}\left(t_{1}, \ldots, t_{p}\right) \sum_{j=1}^{p} \frac{p !}{(j-1) !} \zeta\left(t_{j}, \ldots, t_{p} ; \theta\right) \alpha\left(t_{j}\right),
$$

and

$$
g_{p-1}^{-}\left(t_{1}, \ldots, t_{p-1} ; \theta\right)=\sum_{j=1}^{p} \frac{p !}{(j-1) !} \zeta\left(t_{j}, \ldots, t_{p-1} ; \theta\right)\left\langle f_{p}\left(t_{1}, \ldots, t_{p-1}, \cdot\right), \beta_{t_{j}}(\cdot)\right\rangle,
$$

with $t_{p}=\theta$.

Proof. We will prove the lemma by induction on $p$. Observe that the kernels $g^{+}, g^{\circ}$ and $g^{-}$ are not symmetric functions and that this result uses the convention (2).

For $p=1$, using Proposition 2, we have

$$
\begin{aligned}
I_{1}\left(\beta_{\theta}\right) I_{1}(f) & =I_{2}\left(\beta_{\theta} \circ f\right)+\left\langle\beta_{\theta}, f\right\rangle+\nabla^{+}\left(\beta_{\theta} \phi f\right) \\
& =I_{2}\left(\beta_{\theta} \circ f\right)+\left\langle\beta_{\theta}, f\right\rangle+I_{1}\left(\beta_{\theta} \alpha f\right)+I_{2}\left(\beta_{\theta\left(t_{1}\right)} f\left(t_{1}\right) \circ \beta_{t_{1}}\left(t_{2}\right)\right) \\
& =I_{2}\left(\beta_{\theta} \circ f+\beta_{\theta} f \circ \beta_{t}\right)+I_{1}\left(\beta_{\theta} \alpha f\right)+\left\langle\beta_{\theta}, f\right\rangle,
\end{aligned}
$$

and therefore, in particular, $I_{1}\left(\beta_{\theta}\right) I_{1}(f)$ is in the domain of $\nabla^{+}$.

For the general case, applying Proposition 2 and the fact that $f_{p+1}$ is symmetric, we have

$$
\begin{aligned}
I_{1}\left(\beta_{\theta}\right) I_{p+1}\left(f_{p+1}\right)= & I_{p+2}\left(\beta_{\theta} \circ f_{p+1}\right)+(p+1) I_{p}\left(\left\langle\beta_{\theta}(\cdot), f_{p+1}(*, \cdot)\right\rangle\right) \\
& +(p+1) \nabla^{+}\left(\beta_{\theta}(\cdot) \alpha(\cdot) I_{p}\left(f_{p+1}(*, \cdot)\right)\right) \\
& +(p+1) \nabla^{+}\left(\beta_{\theta}(\cdot) I_{1}(\beta \cdot) I_{p}\left(f_{p+1}(*, \cdot)\right)\right) .
\end{aligned}
$$

Now, applying the induction hypothesis to $I_{1}\left(\beta_{t_{p+2}}\right) I_{p}\left(f_{p+1}\left(*, t_{p+2}\right)\right)$ in the last term, we have,

$$
\begin{aligned}
I_{1}\left(\beta_{\theta}\right) I_{p+1}\left(f_{p+1}\right)= & I_{p+2}\left(\beta_{\theta} \circ f_{p+1}\right) \\
& +(p+1) I_{p+2}\left(\beta_{\theta}\left(t_{p+2}\right) \circ \eta_{p+1}^{+}\left(t_{1}, \ldots, t_{p+1} ; t_{p+2}\right)\right) \\
& +(p+1) I_{p+1}\left(\beta_{\theta}\left(t_{p+1}\right) \alpha\left(t_{p+1}\right) f_{p+1}\left(t_{1}, \ldots, t_{p+1}\right)\right) \\
& +(p+1) I_{p+1}\left(\beta_{\theta}\left(t_{p+2}\right) \circ \eta_{p}^{0}\left(t_{1}, \ldots, t_{p} ; t_{p+2}\right)\right) \\
& +(p+1) I_{p}\left(\left\langle\beta_{\theta}(\cdot), f_{p+1}(*, \cdot)\right\rangle\right) \\
& +(p+1) I_{p}\left(\beta_{\theta}\left(t_{p+2}\right) \circ \eta_{p-1}^{-}\left(t_{1}, \ldots, t_{p-1} ; t_{p+2}\right)\right),
\end{aligned}
$$


where $\eta_{p+1}^{+}(*, r), \eta_{p}^{\circ}(*, r)$ and $\eta_{p-1}^{-}(*, r)$ are the functions defined in the lemma with $f_{p+1}(*, r)$ and $\beta_{r}$ respectively in place of $f_{p}(*)$ and $\beta_{\theta}$.

If we write the kernels of orders $p, p+1$ and $p+2$, we can see that they have the desired form. Note also that we have proved that $I_{1}\left(\beta_{\theta}\right)_{p+1}\left(f_{p+1}\right)$ is in the domain of $\nabla^{+}$.

Let $\Sigma_{p}$ denote the set of all permutations of $\{1, \ldots, p\}$. In the following corollary we replace $f_{p}$ by the product $h_{1} \circ \cdots \circ h_{p}$ to give a partial symmetrization of the kernels obtained in the above proposition.

Corollary 1. If $f_{p}=h_{1} \circ \cdots \circ h_{p}$, where $h_{i} \in L^{2}\left(\mathbb{R}_{+}\right)$for any $i=1, \ldots, p$, we have the following expression:

$$
I_{1}\left(\beta_{\theta}\right) I_{p}\left(h_{1} \circ \cdots \circ h_{p}\right)=I_{p+1}\left(g_{p+1}^{+}(* ; \theta)\right)+I_{p}\left(g_{p}^{\circ}(* ; \theta)\right)+I_{p-1}\left(g_{p-1}^{-}(* ; \theta)\right),
$$

where the symmetrizations of $g_{p+1}^{+}, g_{p}^{\circ}$ and $g_{p-1}^{-}$are given by

$$
\tilde{g}_{p+1}^{+}\left(t_{1}, \ldots, t_{p+1} ; \theta\right)=\sum_{\sigma \in \Sigma_{p}} \sum_{l=0}^{p} \frac{1}{l !} \beta_{t_{l+2}}\left(t_{l+1}\right) \prod_{i=1}^{l} h_{\sigma(i)}\left(t_{i}\right) \prod_{j=3}^{p+2-l}\left(\beta_{t_{l+j}} h_{\sigma(l+j-2)}\right)\left(t_{l+j-1}\right)
$$

with $t_{p+2}=\theta$,

$$
\tilde{g}_{p}^{\circ}\left(t_{1}, \ldots, t_{p} ; \theta\right)=\sum_{\sigma \in \Sigma_{p}} \sum_{l=0}^{p-1} \frac{1}{l !}\left(\alpha \beta_{t_{l+2}} h_{\sigma(l+1)}\right)\left(t_{l+1}\right) \prod_{i=1}^{l} h_{\sigma(i)}\left(t_{i}\right) \prod_{j=3}^{p+1-l}\left(\beta_{t_{l+j}} h_{\sigma(l+j-1)}\right)\left(t_{l+j-1}\right)
$$

with $t_{p+1}=\theta$, and

$$
\tilde{g}_{p-1}^{-}\left(t_{1}, \ldots, t_{p-1} ; \theta\right)=\sum_{\sigma \in \Sigma_{p}} \sum_{l=0}^{p-1} \frac{1}{l !}\left\langle\beta_{t_{l+1}}, h_{\sigma(l+1)}\right\rangle \prod_{i=1}^{l} h_{\sigma(i)}\left(t_{i}\right) \prod_{j=2}^{p-l}\left(\beta_{t_{l+j}} h_{\sigma(l+j)}\right)\left(t_{l+j-1}\right)
$$

with $t_{p}=\theta$.

We are now ready to state the chaotic Kabanov formula.

Theorem 1. Let $g$ be a bounded function with compact support on $\mathbb{R}_{+}$, and

$$
\gamma\left(t_{1}, \ldots, t_{k}\right)=\beta_{t_{2}}\left(t_{1}\right) \cdots \beta_{t_{k}}\left(t_{k-1}\right) g\left(t_{k}\right), \quad t_{1}, \ldots, t_{k} \in \mathbb{R}_{+}, \quad k>1,
$$

and $\gamma(t)=g(t), t \in \mathbb{R}_{+}$, for $k=1$. We have

$$
I_{1}(g) I_{p}\left(f_{p}\right)=I_{p+1}\left(g_{p+1}^{+}\right)+I_{p}\left(g_{p}^{\circ}\right)+I_{p-1}\left(g_{p-1}^{-}\right)
$$

where the kernels $g_{p+1}^{+}, g_{p}^{\circ}$ and $g_{p-1}^{-}$are

$$
g_{p+1}^{+}\left(t_{1}, \ldots, t_{p+1}\right)=\sum_{j=1}^{p+1} \frac{p !}{(j-1) !} \gamma\left(t_{j}, \ldots, t_{p+1}\right) f_{p}\left(t_{1}, \ldots, \hat{t}_{j}, \ldots, t_{p+1}\right),
$$

where $\hat{t}_{j}$ means that $t_{j}$ is omitted in the arguments of $f_{p}$, 


$$
g_{p}^{\circ}\left(t_{1}, \ldots, t_{p}\right)=f_{p}\left(t_{1}, \ldots t_{p}\right) \sum_{j=1}^{p} \frac{p !}{(j-1) !} \gamma\left(t_{j}, \ldots, t_{p}\right) \alpha\left(t_{j}\right)
$$

and

$$
\begin{aligned}
g_{p-1}^{-}\left(t_{1}, \ldots, t_{p-1}\right)= & \sum_{j=1}^{p-1} \frac{p !}{(j-1) !} \gamma\left(t_{j}, \ldots, t_{p-1}\right)\left\langle f_{p}\left(t_{1}, \ldots, t_{p-1}, \cdot\right), \beta_{t_{j}}(\cdot)\right\rangle \\
& +p\left\langle f_{p}\left(t_{1}, \ldots, t_{p-1}, \cdot\right), g(\cdot)\right\rangle .
\end{aligned}
$$

Proof. By Proposition 2, we have

$$
\begin{aligned}
I_{1}(g) I_{p}\left(f_{p}\right)= & I_{p+1}\left(g \otimes f_{p}\right)+p I_{p-1}\left(\left\langle f_{p}(*, \cdot), g(\cdot)\right\rangle\right) \\
& +p \nabla^{+}\left(g(\cdot) \alpha(\cdot) I_{p-1}\left(f_{p}(*, \cdot)\right)\right)+p \nabla^{+}\left(g(\cdot) I_{1}(\beta \cdot) I_{p-1}\left(f_{p}(*, \cdot)\right)\right),
\end{aligned}
$$

and applying Lemma 3 we obtain the decomposition (18).

Proposition 4. Assume now that the underlying martingale is the standard Azéma martingale, that is, $\alpha=0$ and $\beta_{\theta}=-1_{[0, \theta]}, \theta \in \mathbb{R}_{+}$. Then

$$
I_{1}\left(1_{[0, \theta]}\right) I_{p}\left(1_{[0, \theta]}^{\otimes p}\right)=\frac{1}{p+1} I_{p+1}\left(1_{[0, \theta]}^{\otimes(p+1)}\right)-p I_{p-1}\left(\left(t_{(p-1)}-\theta\right) 1_{[0, \theta]}^{\otimes(p-1)}\left(t_{1}, \ldots, t_{p-1}\right)\right),
$$

with $t_{(0)}=0$ and $t_{(p-1)}=t_{1} \vee \cdots \vee t_{p-1}, p>1$.

Proof We let $f_{p}\left(t_{1}, \ldots, t_{p}\right)=\Pi_{i=1}^{p} 1_{[0, \theta]}\left(t_{i}\right)$ and $g=1_{[0, \theta]}$. By Theorem 1 we have

$$
\begin{aligned}
g_{p+1}^{+}\left(t_{1}, \ldots, t_{p+1} ; \theta\right) & =\sum_{j=1}^{p+1} \frac{p !}{(j-1) !}(-1)^{p-j-1} 1_{\left\{t_{j} \leqslant \cdots \leqslant t_{p+1} \leqslant \theta\right\}} \prod_{i=1}^{j-1} 1_{[0, \theta]}\left(t_{i}\right), \\
g_{p}^{\circ}\left(t_{1}, \ldots, t_{p} ; \theta\right) & =0 \\
g_{p-1}^{-}\left(t_{1}, \ldots, t_{p-1} ; \theta\right) & =\sum_{j=1}^{p}(-1)^{p-j} \frac{p !}{(j-1) !} t_{j} 1_{\left\{t_{j} \leqslant \cdots \leqslant t_{p-1} \leqslant \theta\right\}} \prod_{i=1}^{j-1} 1_{[0, \theta]}\left(t_{i}\right),
\end{aligned}
$$

with $t_{p}=\theta$. Symmetrizating and doing some straightforward computations we obtain the desired result.

\section{Consequences of the chaotic Kabanov formula}

In this section we use the chaotic Kabanov formula to prove that the Wiener and Poisson processes are the only normal martingales to possess certain properties relative to polynomials and Wick product. 


\subsection{Existence of a family of orthogonal polynomials associated with the martingale}

Let $X$ be a normal martingale. Following Meyer (1976), we define by induction the martingales

$$
P_{t}^{(0)}=1, P_{t}^{(1)}=X_{t}, \ldots, P_{t}^{(n)}=\int_{[0, t]} P_{s^{-}}^{(n-1)} \mathrm{d} X_{s}, \quad t \in \mathbb{R}_{+} .
$$

Note that $P_{t}^{(n)}=(1 / n !) I_{n}\left(1_{[0, t]}^{\circ n}\right)$ for all $n$. It is well known that in the Wiener case we have the relation $P_{t}^{(n)}=H_{n}\left(X_{t}, t\right)$, where $H_{n}(x, y)=y^{n / 2} h_{n}(x / \sqrt{ } y)$ and $h_{n}$ is the Hermite polynomial of degree $n$.

Also in the Poisson case, we have $P_{t}^{(n)}=C_{n}\left(X_{t}, t\right)$, where $\left\{C_{n}(x, y)\right\}$ are the Charlier polynomials (Meyer 1976; Surgailis 1984).

This situation motivates the following definition:

Definition 3. We will say that a normal martingale $X$ has an associated family of polynomials $\left\{Q_{n}(x, y)\right\}$, where $Q_{n}$ is a polynomial of degree $n$ in $x$, if

$$
P_{t}^{(n)}=Q_{n}\left(X_{t}, t\right), \text { for all } n .
$$

Now the natural problem is to characterize the normal martingales that have an associated family, and the answer is the next theorem.

Theorem 2. Let $X$ be a normal martingale in $L^{4}(\Omega)$. Then $X$ has an associated family of polynomials if and only if $\phi_{s}$ is a deterministic constant process, that is, $X$ is a Poisson or a Wiener process.

Proof. The if part is straightforward. If $\phi_{s}=0$ we are in the Wiener case, and if $\phi_{s}=c \neq 0$, we are in the Poisson case with jumps of height $c$.

The proof of the only if part is a consequence of the Kabanov formula. Assume that the chaotic decomposition of the process $\phi$ is

$$
\phi_{t}=\sum_{n=0}^{\infty} I_{n}\left(f_{n}(\cdot, t)\right)
$$

and $X$ has an associated family of polynomials. Then, by the Kabanov formula,

$$
\begin{aligned}
P_{t}^{(1)} P_{t}^{(1)} & =X_{t}^{2}=I_{1}\left(1_{[0, t]}\right) I_{1}\left(1_{[0, t]}\right)=I_{2}\left(1_{[0, t]}^{\circ 2}\right)+t+\nabla^{+}\left(1_{[0, t]}(x) \phi(x)\right) \\
& =I_{2}\left(1_{[0, t]}^{\circ 2}\right)+t+\sum_{i=1}^{\infty} I_{i}\left(1_{[0, t]}(x) f_{i-1}(\cdot, x)\right) .
\end{aligned}
$$

For each fixed $t, X_{t}^{2}$ will be a linear combination of the three polynomials $Q_{0}\left(X_{t}, t\right)$, $Q_{1}\left(X_{t}, t\right)$ and $Q_{2}\left(X_{t}, t\right)$, which are respectively $1, I_{1}\left(1_{[0, t]}\right)$ and $\frac{1}{2} I_{2}\left(1_{[0, t]}^{\circ 2}\right)$. So

$$
X_{t}^{2}=a_{2}(t) I_{2}\left(1_{[0, t]}^{\circ 2}\right)+a_{1}(t) I_{1}\left(1_{[0, t]}\right)+a_{0}(t) .
$$


Then the chaotic decomposition of $X_{t}^{2}$ has only terms upto the second chaos, and from (19) we obtain $f_{n}=0$ for $n \geqslant 2$. Therefore,

$$
\phi_{t}=f_{0}(t)+I_{1}\left(f_{1}(\cdot, t)\right)
$$

Identifying the kernels of (19) and (20), we have for all $t$

$$
1_{[0, t]}(x) f_{0}(x)=a_{1}(t) 1_{[0, t]}(x)
$$

for the first chaos, and

$$
1_{[0, t]}^{\circ 2}(u, v)+\frac{1}{2}\left(1_{[0, t]}(u) f_{1}(v, u)+1_{[0, t]}(v) f_{1}(u, v)\right)=a_{2}(t) 1_{[0, t]}^{\circ 2}(u, v), \quad u, v \in \mathbb{R}_{+},
$$

for the second chaos. So $f_{0}(u)=c_{0}, u \in \mathbb{R}_{+}$, and $f_{1}(\cdot, x)=c_{1} 1_{[0, x]}(\cdot)$.

Then

$$
\phi_{t}=c_{0}+c_{1} I_{1}\left(1_{[0, t]}\right), \quad t \in \mathbb{R}_{+} .
$$

We now apply Proposition 2 (or the Kabanov formula) to the product $I_{1}\left(1_{[0, t]}\right) I_{2}\left(1_{[0, t]}^{\circ 2}\right)$ with a $\phi$ given by (21), and we obtain

$$
\begin{aligned}
I_{1}\left(1_{[0, t]}\right) I_{2}\left(1_{[0, t]}^{\circ 2}\right)= & I_{3}\left(1_{[0, t]}^{\circ 3}\right)+2 t I_{1}\left(1_{[0, t]}\right) \\
& +2 c_{0} I_{2}\left(1_{[0, t]}^{\circ 2}\right)+\nabla^{+}\left(2 c_{1} 1_{[0, t]}\left(x_{1}\right) I_{1}\left(1_{\left[0, x_{1}\right]}\right) I_{1}\left(1_{[0, t]}\right)\right) .
\end{aligned}
$$

Hence

$$
\begin{aligned}
I_{1}\left(1_{[0, t]}\right) I_{2}\left(1_{[0, t]}^{\circ 2}\right)= & a I_{3}\left(1_{[0, t]}^{\circ 3}\right)+b I_{2}\left(1_{[0, t]}^{\circ 2}\right) \\
& +c I_{1}\left(t 1_{[0, t]}\left(x_{1}\right)+\int_{0}^{t} c_{1} 1_{\left[0, x_{1}\right]}(u) \mathrm{d} u 1_{[0, t]}\left(x_{1}\right)\right),
\end{aligned}
$$

where $a, b, c \in \mathbb{R}$.

As

$$
I_{1}\left(1_{[0, t]}\right) I_{2}\left(1_{[0, t]}^{\circ 2}\right)=X_{t} Q_{2}\left(X_{t}, t\right),
$$

the product is a polynomial of degree 3 in $X_{t}$, and can be expressed as a linear combination of $Q_{0}, Q_{1}, Q_{2}$ and $Q_{3}$. So

$$
\begin{aligned}
\mathrm{I}_{1}\left(1_{[0, t]}\right) I_{2}\left(1_{[0, t]}^{\circ 2}\right) & =b_{3}(t) Q_{3}\left(X_{t}, t\right)+\cdots+b_{0}(t) \\
& =\frac{1}{3 !} b_{3}(t) I_{3}\left(1_{[0, t]}^{\circ 3}\right)+\frac{1}{2 !} b_{2}(t) I_{2}\left(1_{[0, t]}^{\circ 2}\right)+b_{1}(t) I_{1}\left(1_{[0, t]}\right)+b_{0}(t),
\end{aligned}
$$

and as before identifying the kernels of (22) and (23), and focusing our attention to the chaos of order 1, we have

$$
\begin{aligned}
a_{1}(t) 1_{[0, t]}\left(x_{1}\right) & =c\left(t 1_{[0, t]}\left(x_{1}\right)+c_{1} \int_{0}^{t} 1_{\left[0, x_{1}\right]}(u) \mathrm{d} u 1_{[0, t]}\left(x_{1}\right)\right. \\
& =c\left(t 1_{[0, t]}\left(x_{1}\right)+c_{1} x_{1} 1_{[0, t]}\left(x_{1}\right)\right),
\end{aligned}
$$

therefore $c_{1}$ has to be zero, and we get that the process $\phi$ is a constant. 
Remark 3. Since for the standard Azéma martingale we have $\phi_{s}=-I_{1}\left(1_{[0, s]}\right)$, the theorem implies that the Azéma martingale does not have an associated family of polynomials.

Remark 4. In particular we have proved that any normal martingale in $L^{4}$ with an associated family of polynomials is a process with independent increments. In fact, a normal martingale in $L^{4}$ has independent increments if and only if $\phi$ is deterministic (Utzet 1992; Emery 1989).

Remark 5. Yor (1997, Chapter 15) introduces a family of polynomials related to the Azéma martingales in a different context. These polynomials give the conditional expectation of powers for Azéma martingales with respect to the $\sigma$-algebras of the strict past.

\subsection{Projection property for the Wick product}

Let $X$ be a normal martingale in $L^{4}(\Omega)$. The Wick product $I_{1}\left(f_{1}\right): I_{n}\left(g_{n}\right)$ of $I_{1}\left(f_{1}\right)$ and $I_{n}\left(g_{n}\right)$ is defined by

$$
I_{1}\left(f_{1}\right): I_{n}\left(g_{n}\right)=I_{n+1}\left(f_{1} \circ g_{n}\right) .
$$

This motivates the following definition.

Definition 4. We say that $X$ has the Wick projection property if the Wick product $I_{1}\left(f_{1}\right): I_{n}\left(g_{n}\right)$ is the projection of the product $I_{1}\left(f_{1}\right) I_{n}\left(g_{n}\right)$ over the chaos of order $n+1$. Here the product $I_{1}\left(f_{1}\right) I_{n}\left(g_{n}\right)$ is assumed to belong to $L^{2}(\Omega)$ and the kernels $f_{1}$ and $g_{n}$ are bounded and with compact support.

Now the natural problem is to determine the martingales that have this property.

Theorem 3. Assume that $\phi$ has a chaotic decomposition with no terms in chaos greater than 1. Then $X$ has the Wick projection property if and only if $\phi$ is deterministic.

Proof. Proposition 2 says that

$$
\begin{aligned}
I_{1}\left(f_{1}\right) I_{n}\left(g_{n}\right)= & I_{n+1}\left(f_{1} \circ g_{n}\right)+n I_{n-1}\left(\int_{0}^{\infty} f_{1}(s) g_{n}(\cdot, s) \mathrm{d} s\right) \\
& +\nabla^{+}\left(f_{1}(u) \phi(u) n I_{n-1}\left(g_{n}(\cdot, u)\right)\right),
\end{aligned}
$$

and it is straightforward to see that the last addend has null projection over the chaos of order greater than $n$ for all $f_{1}$ and $g_{n}$ if and only if $\phi$ is deterministic.

Remark 6. If in the definition of the Wick projection property we impose $I_{1}\left(f_{1}\right): I_{n}\left(g_{n}\right)$ to be the projection of the product $I_{1}\left(f_{1}\right) I_{n}\left(g_{n}\right)$ over the sum of chaos of order strictly greater than $\mathrm{n}$, instead of over the class of order $\mathrm{n}$, then the above result can be extended to normal martingales $\mathrm{X}$ with $\phi_{t}=\sum_{i} I_{i}\left(f_{i}(\cdot, t)\right)$ for which Proposition 2 is valid. 


\section{Derivation rule of products}

It is well known that on the Wiener space, that is for $\phi=0, \nabla^{-}$is identified with a derivation operator. As noticed by Ma et al. (1998), $\nabla^{-}$cannot act in the same way as a Sobolev derivative, $\nabla^{-} f\left(M_{t}\right)=1_{[0, t]} f^{\prime}\left(M_{t}\right)$, unless $\phi=0$. In this section we study the product rule for $\nabla^{-}$and, in particular, we further show that $\nabla^{-}$can be a derivation operator only for $\phi=0$.

For deterministic $\phi$, Privault (1996) noticed that the product formula becomes

$$
\nabla_{s}^{-}(F G)=F \nabla_{s}^{-} G+G \nabla_{s}^{-} F+\phi_{s} \nabla_{s}^{-} F \nabla_{s}^{-} G, \quad s \in \mathbb{R}_{+} .
$$

This rule does not extend to random $\phi$, but (8) gives by duality the following information.

Proposition 5. For $F, G \in \mathscr{S}$,

$$
\mathrm{E}\left[\nabla_{s}^{-}(F G) \mid \mathscr{F}_{s}\right]=\mathrm{E}\left[F \nabla_{s}^{-} G+G \nabla_{s}^{-} F+\phi_{s} \nabla_{s}^{-} F \nabla_{s}^{-} G \mid \mathscr{F}_{s}\right], \mathrm{d} s \text {-a.e. }
$$

Proof. We write (8) for $u \in \mathscr{U}$ adapted, and apply the duality between $\nabla^{-}$and $\nabla^{+}$:

$$
\begin{aligned}
\mathrm{E} & {\left[\left(u, \nabla^{-}(F G)\right)\right] } \\
& =\mathrm{E}\left[\nabla^{+}(u) F G\right] \\
& =\mathrm{E}\left[G\left(\nabla^{+}(u F)+\left(u, \nabla^{-} F\right)_{L^{2}\left(\mathbb{R}_{+}\right)}+\nabla^{+}\left(u \phi \nabla^{-} F\right)\right]\right. \\
& =\mathrm{E}\left[\left(u, F \nabla^{-} G\right)_{L^{2}\left(\mathbb{R}_{+}\right)}+\left(u, G \nabla^{-} F\right)_{L^{2}\left(\mathbb{R}_{+}\right)}+\left(u, \phi \nabla^{-} F \nabla^{-} G\right)_{L^{2}\left(\mathbb{R}_{+}\right)}\right] .
\end{aligned}
$$

From the above it follows that if $\nabla^{-}$is a derivation then $\mathrm{E}\left[\phi_{s} \nabla_{s}^{-} F \nabla_{s}^{-} G\right]=0, s \in \mathbb{R}_{+}$, $F, G \in \mathscr{S}$, hence $\phi=0$.

As a consequence of Proposition 5, we have

$$
\nabla_{s}^{-}(F G)=F \nabla_{s}^{-} G+G \nabla_{s}^{-} F+\phi_{s} \nabla_{s}^{-} F \nabla_{s}^{-} G+\mathscr{C}_{s}(F, G), \quad s \in \mathbb{R}_{+},
$$

where $\mathscr{C}(F, G)$ is a process with zero adapted projection.

Our aim in the following is to gain more information on this process in the case of the Azéma martingales. We use the notation $\mathscr{C}_{s}(F, t)=\mathscr{C}_{s}\left(F, I_{1}\left(\beta_{t}\right)\right), t \in \mathbb{R}_{+}$.

Proposition 6. Assume that $\phi$ is given as $\phi_{t}=\alpha_{t}+I_{1}\left(\beta_{t}\right), t \in \mathbb{R}_{+}$, where $\alpha$ is locally bounded and $\beta_{t}$ is bounded, $t \in \mathbb{R}_{+}$. Then

$$
\nabla_{s}^{-}\left(I_{1}\left(\beta_{t}\right) F\right)=F \nabla_{s}^{-} I_{1}\left(\beta_{t}\right)+I_{1}\left(\beta_{t}\right) \nabla_{s}^{-} F+\phi_{s} \nabla_{s}^{-} I_{1}\left(\beta_{t}\right) \nabla_{s}^{-} G+\mathscr{C}_{s}(F, t), \quad s \in \mathbb{R}_{+},
$$

where $\mathrm{E}\left[\mathscr{C}_{s}(F, t) \mid \mathscr{F}_{s}\right]=0, s \in \mathbb{R}_{+}$, and $\mathscr{C}_{s}(F, t)$ is given by the relation

$$
\mathscr{C}_{s}(F, t)=\nabla^{+}\left(\beta_{t}(\cdot) \beta_{.}(s) \nabla^{-} F+\phi_{s} \beta_{t}(\cdot) \beta_{.}(s) \nabla^{-} \nabla_{s}^{-} F+\beta_{t} \mathscr{C}_{s}\left(\nabla^{-} F, \cdot\right)\right) .
$$

Proof. We use the relation $\nabla_{s}^{-} \nabla^{+}(u)=\nabla^{+}\left(\nabla_{s}^{-} u\right)+u_{s}$ : 


$$
\begin{aligned}
& \nabla_{s}^{-}\left(I_{1}\left(\beta_{t}\right) F\right) \\
& =\nabla_{s}^{-}\left(\nabla^{+}\left(\beta_{t} F\right)+\left(\beta_{t}, \nabla^{-} F\right)_{L^{2}\left(\mathbb{R}_{+}\right)}+\nabla^{+}\left(\beta_{t} \phi \nabla^{-} F\right)\right) \\
& =\nabla^{+}\left(\beta_{t} \nabla_{s}^{-} F\right)+\beta_{t}(s) F+\left(\beta_{t}, \nabla^{-} \nabla_{s}^{-} F\right)_{L^{2}\left(\mathbb{R}_{+}\right)}+\nabla^{+}\left(\beta_{t} \alpha \nabla^{-} \nabla_{s}^{-} F\right) \\
& +\beta_{t}(s) \alpha_{s} \nabla_{s}^{-} F+\nabla^{+}\left(\beta_{t}(\cdot) \nabla_{s}^{-}\left(I_{1}(\beta .) \nabla^{-} F\right)\right)+\beta_{t}(s) I_{1}\left(\beta_{s}\right) \nabla_{s}^{-} F \\
& =\nabla^{+}\left(\beta_{t} \nabla_{s}^{-} F\right)+\left(\beta_{t}, \nabla^{-} \nabla_{s}^{-} F\right)_{L^{2}\left(\mathbb{R}_{+}\right)}+\nabla^{+}\left(\beta_{t} \alpha \nabla^{-} \nabla_{s}^{-} F\right) \\
& +F \nabla_{s}^{-} I_{1}\left(\beta_{t}\right)+\beta_{t}(s) \alpha_{s} \nabla_{s}^{-} F+\beta_{t}(s) I_{1}\left(\beta_{s}\right) \nabla_{s}^{-} F \\
& +\nabla^{+}\left(\beta_{t}(\cdot)\left(\beta_{.}(s) \nabla_{.}^{-} F+I_{1}(\beta .) \nabla_{.}^{-} \nabla_{s}^{-} F+\phi_{s} \nabla_{2}^{-} I_{1}(\beta .) \nabla_{s}^{-} \nabla_{.}^{-} F+\mathscr{A}\left(\nabla_{.}^{-} F, \cdot\right)\right)\right) \\
& =\nabla^{+}\left(\beta_{t} \nabla_{s}^{-} F\right)+\left(\beta_{t}, \nabla^{-} \nabla_{s}^{-} F\right)_{L^{2}\left(\mathbb{R}_{+}\right)}+\nabla^{+}\left(\beta_{t} \phi \nabla^{-} \nabla_{s}^{-} F\right) \\
& +F \nabla_{s}^{-} I_{1}\left(\beta_{t}\right)+\beta_{t}(s) \alpha_{s} \nabla_{s}^{-} F+\beta_{t}(s) I_{1}\left(\beta_{s}\right) \nabla_{s}^{-} F \\
& +\nabla^{+}\left(\beta_{t}(\cdot) \beta .(s) \nabla_{.}^{-} F+\beta_{t}(\cdot) \phi_{s} \nabla_{s}^{-} I_{1}(\beta .) \nabla_{s}^{-} \nabla^{-} F+\beta_{t}(\cdot) \mathscr{C}_{s}\left(\nabla^{-} F, \cdot\right)\right) \\
& =I_{1}\left(\beta_{t}\right) \nabla_{s}^{-} F+F \nabla_{s}^{-} I_{1}\left(\beta_{t}\right)+\phi_{s} \nabla_{s}^{-} I_{1}\left(\beta_{t}\right) \nabla_{s}^{-} F \\
& +\nabla^{+}\left(\beta_{t}(\cdot) \beta_{.}(s) \nabla_{.}^{-} F+\beta_{t}(\cdot) \phi_{s} \beta_{.}(s) \nabla^{-} \nabla_{s}^{-} F+\beta_{t}(\cdot) \mathscr{C}_{s}\left(\nabla^{-} F, \cdot\right)\right),
\end{aligned}
$$

and we obtain (24).

As a consequence of this proposition, for $F=I_{n}\left(f^{\circ n}\right)$ the remaining process $\mathscr{C}(F, t)$ can be explicitly determined by induction from

$$
\begin{aligned}
\mathscr{C}_{s}\left(I_{n}\left(f^{\circ n}\right), t\right)= & n I_{n}\left(\left(\beta_{t}(\cdot) \beta .(s) f .\right) \circ f^{\circ(n-1)}\right)+n(n-1) f_{s} \nabla^{+}\left(\phi_{s} \beta_{t}(\cdot) \beta .(s) f . I_{n-2}\left(f^{\circ(n-2)}\right)\right) \\
& +n \nabla^{+}\left(f . \beta_{t}(\cdot) \mathscr{C}_{s}\left(I_{n-1}\left(f^{\circ(n-1)}\right)\right), t\right) .
\end{aligned}
$$

Note that $F \mapsto \mathscr{C}(F, t)$ is linear and that the chaotic expansion of $\phi_{s} I_{n-2}\left(f^{\circ(n-2)}\right)$ can be explicitly computed from the Kabanov formula. As an application we compute $\mathscr{A}_{s}\left(I_{n}\left(f^{\circ n}\right), t\right)$ for $n=0,1,2$. We have $\mathscr{A}_{s}(1, t)=0, \mathscr{A}_{s}\left(I_{1}\left(f^{\circ 1}\right), t\right)=I_{1}\left(\beta_{t}(\cdot) \beta .(s) f.\right)$, and

$$
\begin{aligned}
\mathscr{C}_{s}\left(I_{2}\left(f^{\circ 2}\right), t\right)= & I_{2}\left(\beta_{t}(\cdot) \beta .(s) f . \circ f\right)+2 f_{s} \nabla^{+}\left(\left(\alpha_{s}+I_{1}\left(\beta_{s}\right)\right) \beta_{t}(\cdot) \beta .(s) f .\right) \\
& +2 \nabla^{+}\left(f . \beta_{t}(\cdot) I_{1}\left(\beta .(*) \beta_{*}(s) f_{*}\right)\right) \\
= & I_{2}\left(\beta_{t}(\cdot) \beta .(s) f . \circ f\right)+2 f_{s} \alpha_{s} I_{1}\left(\beta_{t}(\cdot) \beta .(s) f .\right)+2 f_{s} I_{2}\left(\beta_{t}(\cdot) \beta .(s) f . \circ \beta_{s}\right) \\
& +2 I_{2}\left(f . \beta_{t}(\cdot) \circ \beta .(*) \beta_{*}(s) f_{*}\right) .
\end{aligned}
$$




\section{Acknowledgements}

We would like to thank Professor Frederic Utzet for fruitful discussions that motivated the problem of the existence of an associated family of polynomials related to a normal martingale. The first named author is grateful for the hospitality of the Centre de Recerca Matemàtica, Barcelona, where this work was completed.

\section{References}

Attal, S. (1998) Classical and quantum stochastic calculus. In L. Hudson and J.M. Lindsay (eds), Quantum Probab. Commun. 10, pp. 1-52. River Edge: World Scientific.

Attal S. and Lindsay J.M. (1997) Quantum stochastic calculus. A new formulation. Preprint.

Biane, P. (1995) Calcul stochastique non-commutatif. In P. Bernard (ed.), Lectures on Probability Theory. École d'Été de Probabilités de Saint-Flour XXIII - 1993, Lecture Notes in Math. 1608. Berlin: Springer-Verlag.

Dellacherie, C., Maisonneuve, B. and Meyer, P.A. (1992) Probabilités et Potentiel. Processus de Markov (fin). Complements de Calcul Stochastique. Paris: Hermann.

Dermoune, A., Krée, P. and Wu, L. (1988) Calcul stochastique non adapté par rapport à la mesure de Poisson. In J. Azéma, P.A. Meyer and M. Yor (eds), Séminaire de Probabilités XXII, Lecture Notes in Math. 1321. Berlin: Springer-Verlag.

Emery, M. (1989) On the Azéma martingales. In J. Azéma, P.A. Meyer and M. Yor (eds), Séminaire de Probabilités XXIII, Lecture Notes in Math. 1372, pp. 66-87. Berlin: Springer-Verlag.

Kabanov, Y.M. (1975) On extended stochastic integrals. Theory Probab. Appl., 20, 710-722.

Ma, J., Protter, P. and San Martin, J. (1998) Anticipating integrals for a class of martingales. Bernoulli, 4, 81-114.

Meyer, P.A. (1976) Un cours sur les intégrales stochastiques. In P.A. Meyer (ed.), Séminaire de Probabilités X, Lecture Notes in Math. 511. Berlin: Springer-Verlag.

Nualart, D. and Vives, J. (1990) Anticipative calculus for the Poisson process based on the Fock space. In J. Azéma, P.A. Meyer and M. Yor (eds), Séminaire de Probabilités XXIV 1988/89, Lecture Notes in Math. 1426, pp. 154-165. Berlin: Springer-Verlag.

Parthasarathy, K.R. (1990) Azéma martingales and quantum stochastic calculus. In R.R. Bahadur (ed.) Proceedings of the R.C. Bose Memorial Symposium, pp. 551-569. Singapore: Wiley Eastern.

Privault, N. (1996) On the independence of multiple stochastic integrals with respect to a class of martingales. C. R. Acad. Sci. Paris, Sér. I, 323, 515-520.

Russo, F. and Vallois, P. (1998) Product of two multiple stochastic integrals with respect to a normal martingale. Stochastic Process. Appl., 73, no. 1.

Surgailis, D. (1984) On multiple Poisson stochastic integrals and associated Markov semi-groups. Probab. Math. Statist., 3, 217-239.

Urbanik, K. (1967) Some prediction problems for strictly stationary processes. In L. LeCam and J. Neyman (eds), Proceedings of the Fifth Berkeley Symposium on Mathematical Statistics and Probability, Vol. 2, pp. 235-258. Berkeley: University of California Press.

Üstünel, A.S. and Zakai, M. (1990) On the structure on independence on Wiener space. J. Funct. Anal., 90, 113-137.

Utzet, F. (1992) Les processus à accroissements indépendants et les équations de structure. In J. Azéma, P.A. Meyer and M. Yor (eds), Séminaire de Probabilités XXVI, Lecture Notes in Math. 1526, pp. 405-409. Berlin: Springer-Verlag.

Yor, M. (1997) Some Aspects of Brownian Motion (Part II). Birkhäuser.

Received October 1997 and revised November 1998 